\title{
Konsistenzüberwachung in Datenbanksystemen - Eine Anforderungsanalyse anhand der Entwurfsbereiche Architektur und Schiffbau
}

\author{
Karol Abramowicz ${ }^{1 \star}$, Birgit Boss ${ }^{1}$, Volkmar Hovestadt ${ }^{2}$, \\ Jutta A. Mülle, Rose Sturm, Peter C. Lockemann ${ }^{3}$ \\ 1 Forschungszentrum Informatik \\ an der Universität Karlsruhe (FZI) \\ Haid-und-Neu-Straße 10-14, 76131 Karlsruhe \\ e-mail: boss@fzide \\ 2 Institut für Industrielle Bauproduktion \\ Fakultät für Architektur und \\ ${ }^{3}$ Institut für Programmstrukturen und Datenorganisation \\ Fakultät für Informatik \\ Universität Karlsruhe, 76128 Karlsruhe \\ e-mail: volkmar@ifib1.ifib.uni-karlsruhe.de, \\ [muelle|sturm|lockemann]@ira.uka.de
}

\begin{abstract}
Zusammenfassung Im technischen Entwurfsbereich können große Teile von Anwendungsprogrammen, die für die Überprüfung der Datenkonsistenz zuständig sind, in das Datenbanksystem verlagert werden. Die Erweiterung von Datenbanksystemen um eine Konsistenzprüfungskomponente ermöglicht eine wesentliche Verkürzung der Überprüfungszeit. Dadurch kann der Datenaustausch zwischen den am Entwurfsprozeß beteiligten Partnern effizienter gestaltet werden. Dieses Papier analysiert anhand von zwei technischen Anwendungsbereichen, Architektur und Schiffbau, vielseitige Anforderungen an Konsistenzprüfungskomponenten in Datenbanksystemen.
\end{abstract}

\section{$1 \quad$ Einleitung}

Immer mehr finden Datenbanksysteme (DBS) Eingang in technische Bereiche, wie CAx-Systeme für Architektur, VLSI-Entwurf, Maschinen- oder Schiffbau, dies nicht zuletzt weil die gewünschte Funktionalität inzwischen nicht mehr durch mangelnde Leistungsfähigkeit behindert wird. Allerdings entstehen aufgrund der in diesen Anwendungsgebieten vorliegenden komplexen Daten und der zwischen ihnen bestehenden Querbezüge hohe Anforderungen nicht nur an eine adäquate Datenmodellierung, sondern auch an die Sicherstellung der Konsistenz. Diese zusätzlichen Anforderungen zu erfüllen, stellt eine besondere und in vielem immer noch neue Herausforderung an die Datenbanktechnik dar, sowohl

\footnotetext{
* inzwischen: AMS Management Systems (Deutschland) GmbH, Am Seestern 1, 40470
} Düsseldorf, e-mail: Karol_W._Abramowicz@mail.amsinc.com 
was die Funktionalität als auch die Leistungsfähigkeit angeht. Ziel des vorliegenden Beitrags ist es, diese Herausforderung deutlich zu machen. Wir wählen dazu zwei Anwendungsfelder, die nach unserer Erfahrung Anforderungen an die Konsistenzhaltung in besonders weitreichender und vielgestaltiger Weise stellen, weil es bei beiden um den Entwurf großer, komplexer Einzelprodukte, also den Einzelentwurf, geht.

Ein Hauptziel der Datenbanktechnik ist es, ein möglichst genaues Abbild der Realwelt in der Datenbank widerzuspiegeln. Ziel der Formulierung von Konsistenzbedingungen ist es, interessierende Sachverhalte der Realwelt, die nicht im Datenmodell erfaßt werden können, ausdrückbar und somit auch vom System abprüfbar zu machen.

Die Integration der Konsistenzbedingungen in das Datenbanksystem bietet viele Vorteile: Sie erzwingt einen einheitlichen Konsistenzbegriff sowohl für alle am Entwurfsprozeß beteiligten Partner als auch für unterschiedliche Werkzeuge. Dies eröffnet die Möglichkeit, daß mehrere Personen oder Werkzeuge koordiniert an einer gemeinsamen Aufgabe arbeiten. Durch die semantisch mächtigeren Ausdrucksmittel der Konsistenzdefinition wird es in einem relativ offenen System, wie es in Entwurfsumgebungen gefordert ist, überhaupt erst möglich, Daten verschiedener Partner gegenseitig nutzbar zu machen. Des weiteren können Konflikte hierbei frühzeitig erkannt und gemeldet werden, was für eine kooperative Arbeitsweise unumgänglich ist. Ein weiterer sehr wichtiger Aspekt ist die durch die Integration ermöglichte Optimierung der Konsistenzprüfung. Wenn die Überwachung der Konsistenz in einem Datenbanksystem stattfindet, läßt sich die Überprüfung auf die Dateneinheiten begrenzen, die nach dem letzten (vom Datenbanksystem) garantierten Konsistenzpunkt modifiziert wurden oder mit diesen in wohl definierter Beziehung stehen. Die Möglichkeit zur deklarativen Beschreibung von Konsistenzbedingungen erlaubt außerdem eine dynamische Anpassung der Überprüfungsstrategie abhängig vom aktuellen Datenbestand. Aus diesen Gründen verspricht die automatische Konsistenzprüfung in Datenbanksystemen für Entwurfsanwendungen eine erhebliche Verkürzung der Entwicklungszeiten und eine Erhöhung der Qualität der Entwürfe.

Die beiden erwähnten Anwendungen entstammen dem Architekturentwurf ${ }^{1}$ und dem Schiffbau ${ }^{2}$. Anhand von Szenarien aus beiden werden im zweiten Kapitel Anforderungen aus Anwendersicht an die Konsistenzunterstützung, deren Gemeinsamkeiten und Unterschiede formuliert. Im darauffolgenden Kapitel werden diese Anforderungen in technische Maßnahmen in Datenbanksystemen übersetzt, und es wird diskutiert, auf welche Mechanismen man sich heute bereits abstützen kann und wo weiterer Forschungsbedarf für die Unterstützung komplexer Konsistenzanforderungen besteht. Eine Zusammenfassung und ein kurzer Ausblick auf unsere laufenden Projekte im Bereich von Konsistenzmechanismen in Datenbanksystemen beschließen den Beitrag.

\footnotetext{
${ }^{1}$ Gefördert im Rahmen des DFG-Projekts "Rechnerintegrierte Gebäudeplanung", Lo/11-1

${ }^{2}$ Gefördert im Rahmen des BMFT-Verbundprojekts ITiS 2b, 18 S0057
} 


\section{Szenarien aus dem Architekturbereich und dem Schiffbau}

\subsection{Gemeinsame Charakteristika}

Bei den betrachteten Anwendungsgebieten handelt es sich wie erwähnt um Einzelproduktionen. Eine Planung wird also jeweils spezifisch für ein Objekt durchgeführt. Hieraus folgt, daß die Planungskosten einen großen Bestandteil der eigentlichen Baukosten pro Objekt ausmachen, während im Gegensatz hierzu die Entwicklungskosten in der Serienproduktion auf sehr viele Objekte verteilt werden können.

Ein weiteres Charakteristikum der Serienproduktion ist, daß mehrere Prototypen, zum Teil im Maßstab 1:1, von dem Produkt erstellt und unterschiedlichen Tests unterzogen werden können, bevor ein Entwurf zur Produktion freigegeben wird. Im Schiffbau und im Architekturbereich ist es dagegen nicht möglich, einen vollständigen Prototyp des künftigen Schiffes bzw. Hauses zu bauen, das Schiff/das Haus selbst ist sozusagen der Prototyp. Die Entwerfer müssen vielmehr fast ausschließlich auf der vorliegenden Erfahrung mit früher gebauten Schiffen und Häusern aufbauen. "Abgemagerte" Prototypen sind allerdings möglich, so etwa im Schiffbau, wo verschiedene Versuche mit verkleinerten Prototypen durchgeführt werden, um z.B. das Strömungsverhalten zu testen. In vielen Bereichen jedoch wie z.B. der Verkehrssicherheit eines Schiffes haben verkleinerte Modelle nur sehr geringe Aussagekraft.

Wie alle Produktbereiche unterliegen auch unsere beiden Anwendungsgebiete einem zunehmenden Zeit-, Qualitäts- und Kostendruck. Ihm versucht man mit Arbeitskonzepten wie der "integralen Planung" zu begegnen [SGKG86, WiHS94]. Die Grundidee der integralen Planung ist es, das Planen zu parallelisieren und zeitlich zu komprimieren, anstatt die einzelnen Gewerke isoliert und sequentiell zu planen und nur deren Ergebnisse aufeinander abzustimmen. Durch die Bildung von interdisziplinären Planungsteams soll die Kommunikation zwischen den Beteiligten verstärkt, sollen frühzeitig Konflikte zwischen verschiedenen Spezialisten erkannt und dadurch bessere und schnellere Lösungen erzielt werden. Dieser Prozeß ist im Schiffbau stärker ausgeprägt als im Architekturbereich. Dies liegt insbesondere an der in weiten Teilen noch in handwerklicher Bautradition begründeten Planungsstruktur in der Achitektur. Jeder Spezialist (Haustechnikingenieur, Bauphysiker, Akustiker, Lichttechniker etc.) führt meist unter der Leitung des Architekten als Treuhänder des Bauherrn und Gesamtkoordinator - die von ihm durchzuführenden Planungsschritte aus, ohne allzusehr auf die Belange der anderen Gewerke Rücksicht zu nehmen. Im Schiffbau ist dies durch einen relativ hohen Industrialisierungsgrad und dem Status der Werft als Generalunternehmen, welches einen Großteil der Planung und Fertigung unter einem Dach vereinigt, anders. So sind sowohl der Einsatz von CAx Systemen, als auch die Ansätze zur Integration von Werkzeugen auf einer gemeinsamen Datenplattform insgesamt im Schiffbau fortgeschrittener als in der Architektur. 
Die These dieses Beitrags ist, daß man den geschilderten Problemen neben einem geeigneten Produktmodell als Basis durch eine Begrenzung der Entwurfsspielräume mittels Konsistenzbedingungen wirkungsvoll begegnen kann. Diese Begrenzung muß allerdings auf gewisse Eigenarten der hier behandelten Entwurfsprozesse Rücksicht nehmen. Sie lassen sich durch deren Nichtlinearität, Vielschichtigkeit und Individualität charakterisieren.

Nichtlinearität Die Nichtlinearität drückt sich in einem ständigen Navigieren zwischen alternativen Lösungen auch auf unterschiedlichen Maßstabsebenen aus. Teile eines Entwurfes können in ihrer Entscheidung zurückgenommen und auf einer anderen Maßstabsebene neu überarbeitet werden. Gerade in größeren Planungsteams entstehen so Teilbereiche der Planung mit unterschiedlicher Bearbeitungstiefe, die die gleiche Wichtigkeit im Gesamtkontext haben. In den verschiedenen Teilen des Entwurfes, die unter anderem räumlich und zeitlich beschrieben werden, können hierbei unterschiedliche Mengen von Konsistenzbedingungen gelten.

Die Menge der Konsistenzbedingungen kann darüberhinaus zeitlichen Änderungen unterliegen. So geht mit der Rücknahme von Entscheidungen eine Veränderung der Menge der Konsistenzbedingungen einher, die für einen bestimmten Entwurfsausschnitt gelten müssen. Kurzfristige Planungsentscheidungen wie die Auswahl eines bestimmten Bauteils oder Materials vor Ort, können ebenfalls Einfluß auf die Konsistenz haben.

Häufig muß man auch bei Zwischenentwürfen noch nicht auf allen Konsistenzbedingungen beharren, d.h. gegenüber dem endgültigen Entwurf können Verletzungen noch toleriert werden, ja Inkonsistenzen sind während des Entwurfsprozesses sogar der Normalfall.

Vielschichtigkeit Jeder am Entwurf Beteiligte hat seine eigene Sicht auf den Entwurf, die geprägt ist durch seine Erfahrungen und seine persönliche Geschichte. Dies äußert sich in einer eigenen Sprache und eigenen Modellen und erschwert so die Kommunikation zwischen den Beteiligten. Diese Vielschichtigkeit stellt an eine Formalisierung des Entwurfes etwa in einer gemeinsamen Datenbank besondere Anforderungen. Die Vielschichtigkeit läßt sich am Beispiel des Schiffbaus veranschaulichen. Bei der Konstruktion werden Teilaspekte (Außenhaut, Stahlkonstruktion, Rohr- und Elektroleitungen) separat entworfen - in einem Schiff müssen sie eine integrale Einheit darstellen.

Individualität Die zumeist unbewußte, unterschiedlich weitgehende Erfahrung aller Beteiligten im Umgang mit Planungsaufgaben im allgemeinen und der Planungsaufgabe im speziellen, ihre auf dieser Erfahrung basierende, nur schwer faßbare Intuition bei der Entscheidungsfindung macht diese im nachhinein für dritte schwer nachvollziehbar und bestimmt in entscheidendem Maße die Individualität und Einmaligkeit des Produktes.

Während die angeführten Anforderungen für beide Anwendungen gleichermaßen gelten, lassen sich doch eine Reihe von unterschiedlichen Merkmalen extrahieren. Diese werden im folgenden ausgeführt. 


\subsection{Architektur}

\section{Charakterisierung des Entwurfsvorgangs}

Ziel Entwurfsziele werden dem Architekten durch den Bauherren aufgrund der geforderten Funktionalität des zu erstellenden Gebäudes vorgegeben. Die Erfüllung dieser Vorgaben ist sehr stark durch die vielfältigen Denkschulen beeinflußt: Ein Architekt versucht immer, eine für ihn ästhetisch zufriedenstellende Lösung zu finden.

Bau- und Planungsumfeld Die Rahmenbedingungen des architektonischen Bauprozesses hängen stark von einem individuell geprägten und meist wenig strukturierten Umfeld ab. Dieses Umfeld wird bei der Planung und Erstellung von Gebäuden im Gegensatz zur Serienproduktion, die auf festgefügte Produktionsstätten und Arbeitsteams zurückgreifen kann, zu großen Teilen für jedes Projekt neu definiert. Es besteht aus einem individuellen Ort, der dort anzutreffenden Infrastruktur und einer Vielzahl unterschiedlichster Bauausführender. Thre Zusammensetzung ist für jedes Gebäude weitgehend unterschiedlich. Sie hängt ab von der Größe, der Art und dem Inhalt des Bauauftrages, sowie dem Ausgang der Ausschreibung der Planungs- und Bauleistungen. Bauträgergesellschaften und Generalunternehmer bilden hier in einigen Bereichen eine Ausnahme.

Die Charakteristika des individuellen Bauumfeldes lassen sich nur in Teilen auf die Zusammensetzung der Planungsteams übertragen. Hier ist der Architekt in der Auswahl der Fachplaner, die zur Planung hinzugezogen werden, relativ frei. So festigen sich über Projektgrenzen hinweg funktionierende Kooperationen, in denen die Kommunikation relativ eingespielt funktionieren kann.

Organisation und zeitlicher Rahmen Der gängige Ablauf der Bauplanung und -ausführung spielt sich innerhalb der Regeln der Baukunst und der gängigen Organisationsformen (VOB, HOAI) ab und läßt sich grob anhand der in der Honorarordnung für Architekten [HOAI91] festgelegten Phasen beschreiben.

Man unterscheidet Grundlagenermittlung, Vorplanung, Entwurfsplanung, Genehmigungsplanung, Ausführungsplanung, Vergabe, Bauausführung und Objektbetreuung mit Dokumentation, die wie Meilensteine die Planung strukturieren und ihr eine Richtung geben. Diese festgefügte Struktur, die die Planungsleistungen vor dem Hintergrund der Honorare regelt, ist immer noch ein großer Hemmschuh für eine weitgehende Parallelisierung der Planungsleistungen innerhalb einer Integralen Planung. Diese würde nämlich zu einer Verwischung der Grenzen zwischen den einzelnen Fachplanern, ihrer Zuständigkeitsbereiche und der allgemeinen Planungsphasen führen.

Automatisierungsgrad Es gibt zwar eine große Menge leistungsfähiger, jedoch spezialisierter und voneinander isolierter Software für die verschiedensten Bereiche des Bauens (CAD, Ausschreibungsprogramme AVA, Kosten- und Energiesimulationsprogramme etc.), aber die Bemühungen im Umfeld integrierter 
Gebäudemodelle mit dem Ziel, eine gemeinsame Datenplattform für die verschiedenen Applikationen zu schaffen, haben bisher noch keinen durchschlagenden Erfolg gehabt (siehe auch [Fisc94]). Deshalb fehlen verläßliche Aussagen zu den Forderungen an Datenbanksysteme hinsichtlich Struktur und Volumen der Daten und Komplexität der Anfragen.

\section{Charakterisierung der Bedingungen}

Funktionale Bedingungen Die funktionalen Bedingungen beginnen mit der Definition der Bauaufgabe an einem bestimmten Ort, der Art des Gebäudes, seiner Funktion, seinem Volumen, den vorgesehenen Kosten, seinem gewünschten Energieverbrauch usw.. Diese Beschreibungen können z.B. in Form eines Pflichtenheftes oder Raumbuchs dem Architekten vorliegen. Darüber hinaus ist die architektonische Planung in ein Beziehungsgeflecht übergeordneter Planung, etwa der Stadt- und Regionalplanung, sowie in Gesetze und Verordnungen eingebunden. Hierzu zählen lokale Bebauungs- und Nutzungsvorschriften (Regionalplan, Flächennutzungsplan, Bebauungsplan mit Geschoßflächenzahl und Grundflächenzahl, Gestaltungssatzung, Denkmalpflege etc.), aber auch allgemeine Bauvorschriften mit Vorschriften zu Abstandsflächen, Fluchtwegen, Feuerwehrzufahrten u.a., die im Bundesbaugesetz oder den Landesbauordnungen geregelt sind.

Physikalische Bedingungen Mit dem Ort kommen weitere zumeist bindende Bedingungen hinzu. So hat jeder Ort eigene individuelle physikalische Bedingungen (Tragfähigkeit des Untergrundes, Höhe des Grundwassers etc.), sowie eine besondere Lage, Besonnung und Erschließung. Zur Erschließung zählen neben der Wegeanbindung der Zugang zu allen Medien wie Wasser, Abwasser, Gas, Elektrizität und Kommunikation.

Konstruktive Bedingungen Bei der Konstruktion eines Gebäudes gelten die sogenannten "Allgemein Anerkannten Regeln der Baukunst". Dies ist ein rechtlicher Begriff, der bei Streitfällen den Maßstab für die "richtige" Konstruktion liefert. Besonderheit ist, daß er nicht notwendigerweise mit dem formalisierbaren Wissen der Baukonstruktionslehrbücher oder den DIN-Normen der einzelnen Gewerke übereinstimmen muß. Die Baukonstruktion entwickelt sich unter dem Druck einer breiten dynamischen Bauindustrie mit einer Vielzahl von Produkten, Materialien und Konstruktionen ständig weiter, so daß das fixierte institutionalisierte Wissen nicht unbedingt auf dem aktuellen Stand ist. Gleichzeitig bedeutet dies, daß alle Konstruktionsweisen im individuellen Baukontext überprüft werden sollten. Das Gleiche gilt für die Bauphysik, die Statik und im Grunde alle anderen Vorgaben, z.B. des Bauherrn, wie Funktions-, Volumen- oder Gestaltvorgaben für das Gebäude. Viele der Bedingungen werden so im Laufe der Entwurfsdiskussionen neu definiert. 
Juristische Bedingungen Die architektonische Planungspraxis ist gekennzeichnet durch eine Vielzahl kleiner individueller Verträge zwischen Auftraggebern, Planern und Ausführenden. Der Architekt und alle Fachplaner fungieren dabei als selbständige Unternehmer mit eigenem Haftungsrisiko, welches in der Regel zeitlich über die Haftung der Ausführenden weit hinausreicht. Dieses persönliche Planungsrisiko, welches durch keine Institution aufgefangen werden kann, wird bewußt und unbewußt zur Grundlage jeder Planungsentscheidung.

Individuelle Bedingungen Schwierigster Teil bei der Beschreibung der Rahmenbedingungen ist das gesellschaftlich kulturelle Umfeld, in dem die Bauaufgabe, der Architekt und der Bauherr stehen. Zu diesem Umfeld gehören kulturelle Aspekte, gestalterische Vorlieben und Ideologien, ein etwaiger Repräsentationsanspruch, die Einbindung in den baugeschichtlichen Kontext o.ä., die durchaus auf Gesetzmäßigkeiten basieren können. Ihre Gewichtung im architektonischen Planungsprozeß ist sehr unterschiedlich. Sie bestimmen mehr oder weniger die Ausprägung des Entwurfes und können je nach Bauaufgabe die übrigen oben beschriebenen Rahmenbedingungen als individuelle Bedingungen überdecken.

Überprüfungszeitpunkte Konsistenzbedingungen sollten im Architekturentwurf selektiv und ereignisgesteuert überprüfbar sein. Wird das Fernziel des "Integralen Planens" angestrebt, so müssen Konsistenzverletzungen frühzeitig an andere am Entwurf Beteiligten weiter geleitet werden können. Falls zum Beispiel zwei Fachplaner den selben Raum widersprüchlich verplanen, müssen sie unmittelbar benachrichtigt werden.

Ein anderer Aspekt ist durch die HOAI vorgegeben: zu bestimmten Meilensteinen müssen bestimmte Bedingungen erfüllt sein, damit der Architekt oder andere Fachplaner ihr Geld einfordern dürfen. Zu diesen Zeitpunkten müssen demnach die entsprechenden Bedingungen überprüft werden.

\section{$2.3 \quad$ Schiffbau}

\section{Charakterisierung des Entwurfsvorgangs}

Ziel Im Schiffbau geht es aufgrund harter zeitlicher Restriktionen und wegen der parallelen Arbeitsweise weniger darum, das "optimale" Schiff zu bauen, als vielmehr ein kostengünstiges, das jedoch trotzdem den Bedingungen z.B. in Hinblick auf die Verkehrssicherheit genügt [GL88]. Die Stahlbleche z.B. müssen bestellt werden, bevor der Optimierungsprozeß abgeschlossen werden kann. Je früher erkannt werden kann, ob eine Anforderung erfüllt ist oder nicht, desto besser für die Qualität des Entwurfs als auch für die Herstellungsdauer.

Bau- und Planungsumfeld Das Bau- und Planungsumfeld im Schiffbau ist im Gegensatz zur Architektur stabiler und stärker strukturiert. Bei der Konstruktion und Herstellung eines Schiffes sind zwar ebenfalls mehrere Partner wie Werften, Ingenieurbüros, Klassifikationsgesellschaften, Versuchsanstalten, Zulieferer und Reeder beteiligt, die sich jedoch von einem Auftrag zum nächsten in ihrer 
Zusammensetzung in der Regel nicht grundlegend ändern. Darüberhinaus sind die Produktionsstätte, die Infrastruktur und die Produktionskapazität definiert, befinden sich größtenteils an einem Ort und sind damit eine berechenbare Größe.

In der Schiffbauindustrie werden fast alle Schiffe als Einzelstücke gebaut, sogar die Schwesterschiffe einer Baureihe unterscheiden sich voneinander durch wesentliche Ausstattungsmerkmale. Immerhin werden jedoch in Versuchsanstalten verschiedene Untersuchungen mit verkleinerten Prototypen durchgeführt, die es erlauben, Teilaspekte der Konstruktion zu überprüfen. Trotzdem spielt Erfahrung eine entscheidende Rolle, so etwa bei einem der wichtigsten Parameter des neuen Schiffes, seiner maximalen Geschwindigkeit. Sie kann nur grob berechnet werden, und auch die Ergebnisse der Tests mit einem verkleinerten Modell in einem Versuchsbecken können nur begrenzt übertragen werden.

Organisation und zeitlicher Rahmen Im Schiffbau ist es üblich, daß aus Termingründen schon in relativ frühen Entwurfszeiten begonnen wird, das Schiff auch wirklich zu bauen. Entwurfsentscheidungen, die sich schon im Produkt niedergeschlagen haben, können damit in der Regel nicht mehr revidiert werden und müssen als harte Bedingungen angenommen werden. Um die extrem kurzen Produktionszeiten (weniger als 18 Monate zwischen dem Vertragsabschluß und der Schiffübergabe) halten zu können, müssen bestimmte Teile und teilweise auch benötigtes Material im voraus bestellt werden. Beispielweise liegt der Bestellvorlauf beim Lieferanten der Hauptmaschine bei rund einem Jahr. Das bedeutet, daß die Hauptantriebparameter geschätzt werden müssen und die Stahlkonstruktion an den Antrieb angepaßt werden muß. Aus diesen Gründen heraus läßt sich im Schiffbau nicht eine so klare Einteilung in verschiedene Phasen finden wie dies in der Architektur der Fall ist.

Automatisierungsgrad Mit CAD-Systemen hat der Schiffbau schon lange Erfahrungen. Zudem hat man frühzeitig die Vorteile effizienter und standardisierter Datenaustauschmethoden erkannt, weil die Partner im Laufe der Zeit an mehreren Projekten zusammenarbeiten, was durch die Marktgegebenheiten (relativ kleine Anzahl der Werften, Zulieferer, Reederei usw.) bedingt ist [FDS91]. Die Leistungsanforderungen kennt man daher recht gut. Allein die Stahlkonstruktion wird durch mehrere hundert Klassen definiert. Für diese Klassen werden normalerweise einige hunderttausend Objekte erzeugt. Das Gesamtdatenvolumen liegt im Gigabyte-Bereich. Aus diesem Grund muß die Konsistenzprüfungskomponente in der Lage sein mit sehr großen Datenmengen effizient zu arbeiten, soll sie den Entwerfer frühzeitig auf mögliche Konsistenzverletzungen hinweisen können. Nur so kann die Qualität des Schiffes garantiert und die Abnahme beschleunigt werden.

\section{Charakterisierung der Bedingungen}

Funktionale Bedingungen Funktionale Bedingungen beschreiben im Schiffbau Bedingungen, die unmittelbar mit dem zu bauenden Schiff zusammenhängen. Es 
kommen, im Gegensatz zur Architektur, nur sehr wenig Bedingungen mit hinzu, die beispielsweise das Umfeld, in dem das Schiff eingesetzt werden soll, beschreiben. Funktionale Bedingungen betreffen z.B. Kostenvorgaben.

Physikalische und konstruktive Bedingungen Im Schiffbau kann nicht zwischen physikalischen und konstruktiven Bedingungen getrennt werden, da sie eng miteinander verknüpft sind. Ein Schiffentwurf oder ein bereits fertiges Schiff muß den Bedingungen z.B. im Hinblick auf die Verkehrssicherheit genügt. Hierunter fällt z.B. die gesamte Statik. Diese Bedingungen können z.B. in EXPRESS formuliert werden. Die entsprechenden Regeln betreffen teilweise das gesamte Schiff, manche nur einzelne Teile.

Juristische Bedingungen Eine Klassifikationsgesellschaft wird von Beginn an in die Planung mit einbezogen, um eine zügige Abnahme des Schiffes zu gewährleisten. Die Abnahme hängt von einer Reihe rechtlich festgelegter Richtlinien und Normen ab, die eingehalten werden müssen. Diese sind stark von dem Land abhängig, in dem das Schiff zugelassen werden soll.

Individuelle Bedingungen In einem Projekt im Schiffbau sind in der Regel eine hohe Anzahl von Personen involviert, die hochgradig parallel arbeiten. An einigen Projekten arbeiten sogar bis zu 70 Personen. Der Entwurf erfolgt auf mehreren Schichten (z. B.: Außenhaut, Stahlkonstruktion, Rohr- und Elektroleitungen), jede der Schichten wird in mehrere Teile aufgesplittet. Ein einzelnes Teil wird von einem Entwerfer auf einmal bearbeitet, wobei sich diese Teile so gut wie überhaupt nicht überlappen. Also lassen sich die Konsistenzbedingungen hier individuell zuordnen, allerdings auf rein objektiver und nicht subjektiver Grundlage.

Überprüfungszeitpunkt Die Personen, die die Konsistenzprüfung vornehmen, sind nicht notwendig identisch mit denjenigen, die das Produkt planen und erstellen. Die Klassifikationsgesellschaft überprüft z.B. das von anderen Partnern entworfene und gebaute Schiff zu bestimmten Meilensteinen. Sie arbeitet schon in frühen Entwurfsphasen relativ eng mit der Werft zusammen, um später eine möglichst schnelle Abnahme des Schiffes zu gewährleisten.

Aber natürlich ist es auch im Interesse der Entwerfer selbst, frühzeitig auf mögliche Konsistenzverletzungen hingewiesen zu werden, insbesondere wenn wie im Schiffbau angesichts der Verzahnung von Planung und Bau Revisionsmöglichkeiten als besonders restriktiv zu betrachten sind. Wichtig dabei ist, daß es nicht genügt, festzustellen, ob eine Bedingung gilt oder nicht, vielmehr sollte auch aufgezeigt werden können, warum eine Bedingung nicht gilt. Nur so kann der Fehler schnell behoben werden.

Die Überprüfung der Bedingungen ist zum Teil sehr zeitaufwendig, und durch die Überprüfung verursachte Arbeitsunterbrechungen sind für die Entwerfer nicht immer zumutbar. Hier sollte der Entwerfer selbst bestimmen können, wann Bedingungen geprüft werden. Beispielsweise könnten sie in "ruhige" Phasen, z.B. nachts, verlegt werden. Das sollte auf Wunsch auch automatisch erfolgen. 
Zusätzlich sollte die Möglichkeit bestehen, bereits zu bestimmten Zwischenzeitpunkten einen gewissen Grad an Konsistenz einzufordern. Hierdurch läßt sich auch die Koordination von kooperierenden Experten leichter unterstützen. Im Schiffbau wird man wegen der fehlenden Revisionsmöglichkeiten sogar Zeitpunkte vorgeben müssen, zu denen bestimmte Konsistenzbedingungen erfüllt sein müssen, während andere durchaus noch offengelassen werden können.

\section{Anforderungen an einen Konsistenzmechanismus}

In diesem Kapitel werden nun die konkreten Anforderungen an einen Konsistenzprüfungsmechanismus in den oben vorgestellten Anwendungen beschrieben. Hierbei werden die einzelnen Anforderungen aufgestellt und jeweils durch die Anwendungen begründet. Anforderungen generell an Datenbanksysteme in Nicht-Standard-Anwendungen, um welche es sich bei den beiden hier betrachteten Bereichen handelt, wurden schon in zahlreichen Arbeiten der 80er Jahre, wie z.B. [LAB+85], behandelt. Jedoch gibt es bis heute insbesondere für den Bereich der Konsistenz keine umfassend zufriedenstellenden Lösungen. Teile dieser Anforderungen werden in Veröffentlichungen zu aktiven Datenbanksystemen behandelt, z.B. [AnMC93, BBKZ93, DiPG91, GaDi94a, GeJa91, Hans92, Jasp94, KoDM88, McDa89, SPAM91, StHP91, Wido92], einen vollständigeren Überblick geben [Jaeg94, Baye93, IEEE92, VoKe91, KiLS91]. Der Themenkreis wird ebenfalls in Wissensbankverwaltungssystemen (KBMS) (einen Überblick gibt z.B. [BoDa93]) behandelt. Bei der Beschreibung der Anforderungen gehen wir hauptsächlich auf die Aspekte näher ein, die unserer Meinung nach bislang noch nicht in ausreichendem Maße betrachtet wurden. Die Anforderungen werden jeweils durch Beispiele aus den von uns konkret betrachteten Anwendungsgebieten Architektur und Schiffbau veranschaulicht.

\subsection{Sprachmittel zur Konsistenzbeschreibung}

Bei der Betrachtung von Sprachmitteln für technische Bereiche müssen verschiedene Aspekte abgedeckt werden. Zum einen spielen Ausdrucksmächtigkeit und Formulierbarkeit durch den Benutzer eine wichtige Rolle, zum anderen sind Aspekte wie die entwurfsausschnittsbezogene und zeitliche Lokalität der Konsistenzbedingungen und Standardisierungsbemühungen von Bedeutung.

Ausdrucksmächtigkeit: Aufgrund der Komplexität der Anwendungsgebiete ist eine Vielzahl der verschiedensten Arten von Konsistenzbedingungen abzudecken. Neben sehr konkreten Angaben, wie etwa der maximalen Spannweite eines Trägers, existieren auch sehr vage Bedingungen, z.B. daß “das Kinderzimmer sehr groß und hell sein soll".

Um dieser Ausdrucksmächtigkeit Herr zu werden, ist die Unterstützung sowohl von deklarativen als auch von prozeduralen Konsistenzbedingungen notwendig. Deklarative Bedingungen sind im Anwendungsgebiet der Architektur beispielsweise die Gleichheit von Bauteilen oder auch Beziehungen der Form, 
wann ein Objekt neben einem anderen Objekt liegen soll. Prozedurale Bedingungen eignen sich demhingegen zur Beschreibung von komplizierteren Zusammenhängen wie Restriktionen, die die Luftaustauschrate eines Raumes erfüllen muß. Meistens wird eine Eigenschaft, die der fertige Entwurf haben soll, nicht durch einen einzelnen Wert beschrieben, sondern durch eine Menge von möglichen Werten, wobei jedoch gewisse Präferenzen zwischen den Werten bestehen. In der natürlichen Sprache sind solche Präferenzen implizit in vagen Begriffen wie "groß" etc. enthalten. Zusätzlich muß Unsicherheit bzgl. einer Bedingung ausgedrückt werden können, bzw. der Grad, zu dem man Abweichungen von der Bedingung tolerieren kann. In der natürlichen Sprache drücken wir solche Unsicherheiten mit Worten wie "soll", "muss", "sollte" aus. Da viele Konsistenzbedingungen als Entwurfsentscheidungen überhaupt erst im Laufe des Entwurfes entstehen und deshalb durch den Entwerfer selbst in das System eingebracht werden können sollen, muß sie oder er selbst die Ausdrucksform wählen können.

Eine vollständige Umsetzung dieser Anforderungen in einem Datenbanksystem ist uns nicht bekannt. EXPRESS [EXPR94], als Standardisierungsansatz, unterstützt im Ansatz die Formulierung sowohl von deklarativen als auch von prozeduralen Bedingungen. Allerdings ist hier die direkte Integration in die Datenbank noch nicht gelöst; die in EXPRESS formulierbaren Bedingungen sind so weit gefaßt, daß eine automatische Umsetzung in existierende Systeme nicht erfolgen kann, sondern entsprechende zusätzliche Programmierung erforderlich wird. Zur Unterstützung vager und unsicherheitsbehafteter Konsistenzbedingungen gibt es bislang kaum Arbeiten [Boss94].

Gültigkeitsbereich: Zusammen mit der Formulierung einer Konsistenzbedingung ist es notwendig, ihren Gültigkeitsbereich festzulegen. Gerade die vom Entwerfer dynamisch eingebrachten Bedingungen gelten nur für genau den durch den Benutzer definierten Entwurfsausschnitt und nicht für den gesamten Entwurf bzw. alle Objekte entsprechenden Typs. Da es für Entwurfsaufgaben typisch ist, daß Entwürfe in verschiedenen Ausschnitten unterschiedlich weit gediehen sind, gelten in den unterschiedlichen Ausschnitten aber auch unterschiedliche Mengen von Konsistenzbedingungen. Gefordert ist also eine sehr flexible Zuordnung von Entwurfsbedingungen zu spezifizierten Ausschnitten aus dem Gesamtentwurf.

Gültigkeitsbereiche können neben Entwurfsausschnitten natürlich auch einzelne Objekte oder Objekttypen sein. So muß es z.B. möglich sein, Restriktionen, die beispielsweise alle Stützen in einem Gebäude beschreiben, dem entsprechenden Objekttyp zuzuordnen. Andere Bedingungen dagegen betreffen genau einen Tisch, einen Raum oder die Brücke eines Schiffs.

Existierende Ansätze erlauben es i.d.R., Konsistenzbedingungen sowohl für einzelne Objekte als auch für Objekttypen zu formulieren. Nicht unterstützt wird eine Einschränkung des Gültigkeitsbereichs auf beliebige Ausschnitte des Gesamtentwurfs unabhängig von einer Objekt- bzw. Typbindung.

Formulierbarkeit durch den Benutzer: Ein Teil der Konsistenzbedingungen stellen einen integralen Bestandteil des Entwurfes dar und sind als Entwurfsentscheidungen aufzufassen. Deshalb muß es möglich sein, diese Bedingungen dynamisch 
zur Laufzeit in das DBS einfügen zu können. Das heißt u.a. auch, daß nicht eine speziell ausgebildete Person, z.B. der Datenbankadministrator, die Bedingungen formuliert, sondern die Entwerfer selbst. Dies stellt hohe Anforderungen an die Verständlichkeit der Sprache zur Formulierung der Bedingungen.

Wie schon zuvor beschrieben, müssen sowohl präzise als auch vage und unsichere Bedingungen beschrieben werden können. In beiden Fällen muß es dem Benutzer ermöglicht werden, möglichst intuitiv seine Bedingungen zu formulieren. Gerade bei den vagen Bedingungen wäre hier ein Ansatz wünschenswert, der dem Nutzer die Möglichkeit gibt, Bedingungen mithilfe von linguistischen Begriffen, die dem Entwerfer vertraut sind, zu formulieren. Ein solcher Ansatz könnte z.B. auf der Theorie der Fuzzy-Mengen basieren. In keinem der uns bekannten Ansätze ist es möglich, Bedingungen mittels linguistischer Begriffe in Datenbanken zu definieren. Wünschenswert wäre weiter eine Unterstützung durch adäquate Benutzeroberflächen und vordefinierte Sprachelemente.

Die meisten Ansätze legen den Schwerpunkt auf die Definition von globalen Bedingungen, d.h. Bedingungen, die zu Beginn einer Anwendung oder zur Schemadefinitionszeit definiert werden. Der Aspekt des dynamischen Definierens, Änderns und Entfernens von Bedingungen bzw. Bedingungsmengen steht in der Regel nicht im Mittelpunkt der Betrachtung, auch wenn dies zum Teil vom Konzept her möglich wäre. Entsprechend wird auch der Aspekt der benutzerabhängigen Prioritäten von Bedingungen nicht betrachtet. (Dagegen unterstützen mehrere Systeme global gültige Prioritäten zwischen einzelnen Regeln [AgCL91].)

Dem Anwender müssen auch Mittel an die Hand gegeben werden, die Gültigkeitsbereiche seiner Konsistenzbedingungen beliebig selber festzulegen. Hierbei ist es durchaus üblich, daß jede Konsistenzbedingung ihren eigenen Gültigkeitsbereich hat. Damit einher geht die Notwendigkeit, Konsistenzbedingungen explizit aktivieren und deaktivieren zu können, dies wiederum bezogen auf einen bestimmten Ausschnitt des Entwurfs.

Standards: Immer mehr gewinnen Standards Einfluß auf die verschiedenen Anwendungsdomänen. So wurden ein Teil der Konsistenzregeln im Schiffbau bereits in EXPRESS formuliert [IGR93]. Dementsprechend entsteht auch die Forderung, Konsistenzregeln, die in bestehenden Standards beschrieben sind, in ein Datenbanksystem zu integrieren.

\subsection{Zeitpunkt der Überprüfung}

In existierenden Ansätzen werden durchaus schon mannigfaltige Mechanismen zur Unterstützung flexibler Zeitpunkte zur Überprüfung von Konsistenzbedingungen angeboten, z.B. [CKAK94, GeJS92, GaDi94b]. Bezüglich des im Entwurf wichtigen Koordinations- und Kooperationsaspekts gibt es allerdings erst in jüngerer Zeit Arbeiten zu kooperierenden Transaktionen, z.B. [Duer94, JBK+93].

Bei einer wachsenden oder großen Anzahl von Bedingungen ist es sehr schwer für den einzelnen Entwerfer, den Überblick darüber zu behalten, welche Bedingungen zu welchem Zeitpunkt erfüllt sein müssen. Dies gilt noch verstärkt, wenn 
sie oder er den Zeitpunkt der Überprüfung selbst bestimmt. Hier sind Hilfestellungen z.B. in Form von Browsern nötig, um Informationen zu erhalten, welche Bedingungen zu welchem Zeitpunkt überprüft werden sollten. Eine gewisse Automatisierung ließe sich durch Einsatz von Methoden des Workflow Management und die Möglichkeit des Erkennes komplexer Ereignisse, die den Eintritt in eine neue Entwurfsphase einläuten, denken. Sie könnten auch dazu benutzt werden, Vorhersagen über die potentiell abzuarbeitenden Konsistenzbedingungen zu machen. Damit werden Vorarbeiten zur Sicherstellung einer schnellen Konsistenzprüfung möglich. Weiter könnte der Entwerfer in seiner Arbeit unterstützt werden, wenn Konsistenzbedingungen, von denen bekannt ist, daß sie in einem bestimmten Zustand erfüllt sein sollten, zu diesem Zustand automatisch aktiviert bzw. aufgerufen und abgearbeitet werden. Voraussetzung ist die Möglichkeit einer ereignisgesteuerten Überprüfung von Konsistenzbedingungen, wobei die Ereignisse sehr komplex sein können.

\subsection{Partielles Rücksetzen und Revidieren von Entwürfen bzw. Entwurfsentscheidungen}

Durch die Nichtlinearität des Entwurfs kommt es häufig vor, daß Entwurfsentscheidungen neu überdacht werden müssen. Manchmal möchte man in diesem Fall ganz konkret auf einen bestimmten Zustand zurücksetzen und noch einen Versuch starten. Im anderen Fall möchte man eine neue Entscheidung in das System einbringen, von der man weiß, daß sie eventuell zu anderen bereits zuvor getroffenen Entscheidungen in einem gewissen Konflikt stehen könnte. Diese müssen identifiziert und entsprechend modifiziert bzw. entfernt werden. Diesen beiden Fällen entsprechend unterscheiden wir zwischen Rücksetzen und Revidieren.

Rücksetzbarkeit: Es muß die Möglichkeit geben, einen Entwurf in einen beliebigen Ausschnitt eines beliebigen Zeitpunkts in der Vergangenheit zurückzusetzen, also alle Entwurfsentscheidungen in diesem Ausschnitt entsprechend zurückzunehmen. Wird nun davon ausgegangen, daß im Laufe des Entwurfes immer mehr Konsistenzbedingungen dynamisch zugeschaltet werden, so müssen diese ebenfalls mit zurückgesetzt werden. Es ergeben sich die folgenden Probleme für eine Konsistenzüberwachung :

- Für den zurückgesetzten Teil muß wiederum die Menge der Konsistenzbedingungen lokalisiert werden, die zu diesem Zeitpunkt in dem entsprechenden Bereich aktiviert waren. Da Inkonsistenzen zugelassen werden, ist auch in bezug auf diesen Aspekt auf den alten Zustand zurückzusetzen.

- Da andere Entwurfsausschnitte im neuen Zustand beharren, also der Gesamtentwurf nur partiell zurückgesetzt wurde, ist mit einer Fülle neuer Konsistenzverletzungen zu rechnen.

In bisherigen Ansätzen ist Rücksetzbarkeit immer mit Versionierung oder mit dem Begriff der langen Transaktionen verbunden. Im ersten Fall, der expliziten 
Versionierung, gibt der Anwender an, in welchem Ausschnitt und zu welcher Zeit sie oder er eine bestimmte Version festlegen will. Im Rahmen von langen Transaktionen kann entweder auf den Zustand zu Beginn der Transaktion oder aber zu definierten Sicherungspunkten zurückgesetzt werden. Dieser Ansatz ist relativ flexibel, erlaubt das Zurückzusetzen allerdings nur innerhalb einer Transaktion und nicht über Transaktionsgrenzen hinaus. Beide Ansätze haben sich bisher nicht damit befaßt wie mit nicht lokalen Konsistenzbedingungen umgegangen werden soll.

Revidieren: Das Ziel einer Revision ist, daß möglichst wenig Änderungen in Bezug auf die Gesamtmenge der bereits getroffenen Entscheidungen bzw. Bedingungen vorgenommen werden müssen, die neu eingebrachte Bedingung jedoch nach der Änderung im System enthalten ist, also nicht zurückgewiesen wird, die Menge der Bedingungen nicht in sich inkonsistent ist und daß außerdem die Datenbasis mit diesen Bedingungen konform ist. Bedingungen und Daten also, die in einem nicht tolerierbaren Grad in Konflikt mit der neuen Bedingung stehen, müssen gelöscht bzw. entsprechend modifiziert werden. Ein Beispiel für eine Revision ist die Entscheidung, doch kein Tropenholz zu verwenden: Alle Entscheidungen für Verschalungen, die solches verwenden, werden durch diese neue Entscheidung hinfällig. Andere Entscheidungen werden jedoch davon nicht berührt. Eine Revision ist nicht immer erfolgreich, z.B. dann nicht, wenn Bedingungen höherer Priorität im System enthalten sind, die in Konflikt mit der neuen Entscheidung des Entwerfers stehen.

Das Revidieren von Entwurfsentscheidungen wird in bisherigen Mechanismen zur Überprüfung der Konsistenz nicht berücksichtigt. Das dürfte zu einem großen Teil daran liegen, daß die meisten Ansätze eher Bedingungen betrachten, die für sämtliche Entwürfe gelten, als solche, die nur für einen einzelnen Entwurf gültig sind. Aber selbst bei ersteren muß mit Revisionen von Zeit zu Zeit (wenn auch in größeren zeitlichen Abständen) gerechnet werden, insbesondere dann, wenn sich das Schema ändert.

\subsection{Reaktion auf Konsistenzverletzung}

Wie schon erwähnt, werden Inkonsistenzen bestimmter Regeln oder Regelmengen in bestimmten Entwurfsphasen bewußt in Kauf genommen. Dies ist unabhängig davon, ob eine Bedingung präzise oder vage formuliert oder von vornherein mit Unsicherheiten behaftet ist. Ein einfaches Deaktivieren der betroffenen Bedingungen in solchen Entwurfsphasen ist jedoch nicht immer ausreichend, da Reaktionen durchaus erwünscht sein können. Grob unterscheiden wir zwischen "harten" und "weichen" Konsistenzbedingungen, je nachdem ob die entsprechende Bedingung erfüllt sein muß oder Inkonsistenzen toleriert werden. Da dies von der Entwurfsphase abhängig ist, muß man eine Bedingung beliebig von "weich" auf "hart" und umgekehrt schalten können. Angenommen, ein bestimmter Raum solle 2 Türen haben. Zu Beginn der Planung ist diese Bedingung weich, da sie für den Architekten lediglich eine Art Merkfunktion darstellt. In der Phase, in der der Architekt diesen Raum genau spezifiziert, wird diese Bedingung 
hart und bleibt es auch, es sei denn der Entwerfer stößt einen Revisionsprozeß an oder setzt den Entwurf wieder zurück.

$\mathrm{Ob}$ eine Bedingung hart oder weich ist, hat Auswirkungen auf die mögliche Reaktion auf eine Verletzung. Bei harten Bedingungen wird erwartet, daß die Daten, die die Konsistenzverletzung verursachen, nicht so in den Entwurf aufgenommen werden. Bei weichen Bedingungen dagegen ist eine umfangreiche Palette möglicher Reaktionen denkbar. Dies reicht vom einfachen Warnen des Entwerfers, daß die Bedingung verletzt ist, bis zum Starten eines aufwendigen Kommunikationsprozesses, wie die Unstimmigkeiten behoben werden können, in den alle betroffenen Projektpartner einbezogen werden. Wenn Entwerfer Konsistenzverletzungen selbst beheben sollen, reicht das einfache Anzeigen, welche Bedingung mit welchen Belegungen verletzt ist, jedoch meist nicht aus. Vielmehr müssen auch die Ursachen dafür angegeben werden, soweit diese vom DBS erkannt werden können. Im Idealfall werden dem Entwerfer sogar Vorschläge für Reparaturen gemacht [MoLo91, Frie93].

Die Unterteilung in "Bedingung erfüllt" oder "Bedingung nicht erfüllt" ist sehr grob. Sie unterscheidet nicht zwischen Werten, die die Bedingung "fast" erfüllen und solchen, die sie mit Sicherheit nicht erfüllen. Ideal wäre eine weitere Unterteilung in verschiedene Grade der Konsistenz bzw. Inkonsistenz.

Die unterschiedlichen geforderten Reaktionen auf die Verletzung von Konsistenzbedingungen können teilweise durch aktive Datenbanken modelliert werden. Hier muß dann der entsprechende Implementierungsaufwand aufgebracht werden. Ein System, in dem auch explizit die Möglichkeit unterstützt wird, daß harte zu weichen Constraints werden und umgekehrt, wird z.B. in [EiWe93] diskutiert. Es gibt kaum Ansätze, die verschiedene Grade von Inkonsistenz unterscheiden: entweder wird (bezogen auf eine Regel) jegliche Inkonsistenz toleriert, oder gar keine. Manchmal wird der tolerierte Grad der Inkonsistenz oder die relative Konsistenz durch zeitliche Bedingungen und die Menge der sich in einem bestimmten Zeitraum geänderten Daten beschrieben [RuSK91, Datt94].

\subsection{Leistung}

Die Abarbeitung von Konsistenzbedingungen kann zum Teil recht zeitaufwendig werden, was abhängig ist von der Menge der Bedingungen, die zu prüfen sind, der Größe der Datenbasis bzw. der Menge der zu überprüfenden Objekte, sowie von der Komplexität der Bedingungen selbst. Die Leistungsanforderungen unterscheiden sich auch je nachdem, ob die Bedingungen zu einem bestimmten Zeitpunkt einmalig überprüft werden oder ständig im Hintergrund überwacht werden sollen. Im Hintergrund überwachte Bedingungen dürfen den Gesamtfluß des Entwurfs möglichst wenig beeinträchtigen. Bei einmaligen Überprüfungen kommt es nicht zuletzt auf die subjektive Einschätzung der Komplexität und der Gewichtung der Bedingungen durch den Entwerfer an, welche Wartezeiten in Kauf genommen werden. Für Bedingungen, für die gegenwärtig eine Person tagelang Berechnungen ausführt, bietet sich kaum eine Alternative zum System an, selbst wenn auch dort eine stundenlange Berechnung anfällt. Teilweise können 
Verletzungen einer solchen Bedingung mit einem Blick auf eine Zeichnung schneller erkannt werden, jedoch ist bei dieser Vorgehensweise nicht gewährleistet, daß wirklich alle Fehler entdeckt werden. Bei wichtigen Bedingungen ist das nicht hinnehmbar, bei anderen dagegen wie z.B. der Festigkeitsprüfung ist eine intuitive Prüfung anhand der Zeichnung manchmal ausreichend. Folgt man etwa einem Workflow-Ansatz gemäß Abschnitt 3.2, so wird man daher unterscheiden wollen, ob eine systemrealisierte Überprüfung erfolgen soll oder der Entwerfer zu einer intellektuellen Überprüfung aufgefordert wird.

Verschiedene Arbeiten haben sich mit Leistungsaspekten befaßt. Einige beschäftigen sich mit der effizienten Abarbeitung einer kompletten Regelmenge (z.B. durch DB-adäquate Variationen des Rete-Algorithmus [EiWe93, FaRS93]), durch adäquate Transaktionsmechanismen, z.B. [DaRa93, SrHT90], oder durch algebraische Optimierung [Herz94], andere mit der effizienten Ausführung einer einzelnen Regel, z.B. [QiSm93, Alt94]. Insgesamt stellt aber bis heute das Problem der Leistungsoptimierung das größte Hindernis für einen breiten und flexiblen Einsatz von Konsistenzbedingungen dar.

\section{Zusammenfassung und Ausblick}

Der Beitrag ging von der These aus, daß Konsistenzbedingungen ein wesentliches Mittel sein können, um im Entwurfsprozeß Entscheidungen zu dokumentieren und in der Datenbasis durchzusetzen. Wir behaupteten darüberhinaus, daß diese Entscheidungen und damit die Bedingungen besonders hohe Ansprüche an ein Datenbanksystem stellen, wenn es sich um den Entwurf von Einzelprodukten handelt. Um diese Thesen zu belegen, untersuchte der Beitrag zunächst zwei Anwendungsfelder, in denen Einzelentwürfe dominieren. Diese Untersuchungen erfolgten ursprünglich völlig unabhängig voneinander. Die Tatsache, daß sich die jeweils gemachten Beobachtungen so stark überdeckten, war ein wesentliches Motiv für den vorliegenden Beitrag.

Es zeigte sich, daß derartige Anwendungen eine Vielfalt zum Teil recht ungewohnter Anforderungen an die Beschreibung und Überprüfung von Konsistenzbedingungen stellen, die u.a. die Präzision der Formulierung, die Gültigkeitsbereiche und -zeitpunkte, die Überprüfungszeitpunkte, die Reaktion auf Verletzungen, das dynamische Einbringen, Revidieren und das Rücksetzen betreffen. Ein kurzer Vergleich mit dem Stand der Technik machte deutlich, daß die Umsetzung der Forderungen an Datenbanksysteme noch zahlreiche neuartige Fragestellungen aufwirft.

Einige dieser Fragestellungen verfolgen wir derzeit in zwei Vorhaben. Das Projekt ArchE soll ein Framework zur Verfügung stellen, das den architektonischen Entwurfsprozeß von Beginn an, also schon bei der Erstellung der ersten Anforderungsanalysen, bis zum Gebäudeabriß unterstützt [HFH+93, LMSH94]. Insbesondere richten wir hierbei unser Augenmerk jedoch auf die frühen noch sehr schwach formalisierbaren Entwurfsphasen und auf das Ziel, die dort anfallenden Daten für Experten (Fachplaner [HoMS94, StLo94]), die erst in weiteren Entwurfsphasen hinzukommen, zu integrieren. Als Testumgebung für die- 
se Aufgabenstellung haben wir die Fachplaner MIDI und Armilla ausgewählt. MIDI ist ein Stahlbausystem, das den Bau von mehrgeschossigen, hochinstallierten Gebäuden erlaubt. Armilla ist das hierzu passende allgemeine Installationsmodell, ein Regelbuch für die koordinierte Installationsführung in Gebäuden [Hall74, Hall85].

Ziel des ITiS-Projektes ist die Bereitstellung von Konsistenzprüfungsmechanismen für objektorientierte Datenbanksysteme im Schiffbau unter Verwendung der STEP/EXPRESS-Norm. Die Konsistenzbedingungen sowie Schemata sind in EXPRESS beschrieben. Ein besonderes Augenmerk wird auf die effiziente Bedingungsauswertung gelegt, da die Zeit einen besonders kritischen Faktor darstellt.

\section{Danksagung}

Wir möchten den anonymen Gutachtern für ihre zahlreichen Kommentare zu der ersten Version dieses Beitrages danken. Des weiteren danken wir unseren Kollegen Jochen Alt, Dr. Günter von Bültzingsloewen, Uwe Herzog und Dr. Ralf Kramer für weitere Anregungen und Korrekturen.

\section{Literatur}

[Alt94] J. Alt, Optimizing EXPRESS Rule Evaluation with Fine-grained, Dynamic Materialization in CAD Applications, Proc. 4th Int. Conf. of EXPRESS Users Group, Greenville, South Carolina, Oct. 1994.

[AgCL91] R. Agrawal, R.J. Cochrane, B.G. Lindsay On Maintaining Priorities in a Production Rule System, Proc. of 17th VLDB, Barcelona, 479-487, Sept. 1991.

[AnMC93] E. Anwar, L. Maugis, S. Chakravarthy A New Perspective on Rule Support for Object-Oriented Databases, Proc. Int. Conf. on Management of Data, Washington D.C., 99-108, 1993.

[Baye93] P. Bayer State-of-the-art report on reactive processing in databases and artificial intelligence, The Knowledge Engineering Review, Vol. 8:2, Cambridge University Press, 145-171, 1993.

[BBKZ93] H. Branding, A. P. Buchmann, T. Kudraß, J. Zimmermann Rules in an Open System: The REACH Rule System, Proc. 1st Int. Workshop on Rules in Database Systems, Edinburgh, 111-126, Aug. 1993.

[BoDa93] B. Boss, C. Danner Rule Management in Expert Database Systems, ESPRIT Project 736, Technical Report JCF/FZI/004-04/13-Feb-93, Feb. 1993.

[Boss94] B. Boss Handling of Vague and Uncertain Design Constraints in an Object Oriented Database, ESPRIT Project 7364, Technical Report JCF/FZI/039-01/15-Nov-94, Nov. 1994.

[CKAK94] S. Chakravarthy, V. Krishnaprasad, E. Anwar, S.-K. Kim Composite Events for Active Databases: Semantics, Contexts and Detection, Proc. of 20th VLDB, Santiago, Chile, 606-617, Sept. 1994.

[DaRa93] C. Danner, M. Ranft Transaction Management to Support Rule Based Database Applications, Proc. 1st Int. Workshop on Rules in Database Systems, Edinburgh, 143-162, Aug. 1994. 
[Datt94] A. Datta Research Issues in Databases for ARCS: Active Rapidly Changing Data Systems SIGMOD RECORD, Vol. 23, no. 3, 8-13, Sept. 1994.

[DiPG91] O. Diaz, N.W. Paton, P. Gray Rule Management in Object Oriented Databases: A Uniform Approach, Proc. of 17th VLDB, Barcelona, 317-326, Sept. 1991.

[Duer94] M. Dürr Koordinationsmechanismen für Teamarbeit: Modellbildung und Datenbank-Unterstützung, VDI-Fortschrittsberichte, Reihe 10, Nr. 296, 1994.

[EiWe93] C.F. Eick, P. Werstein Rule-Based Consistency Enforcement for Knowledge-Based Systems, IEEE Transactions on Data and Knowledge Engineering, Vol. 5, 52-64, Feb. 1993.

[EXPR94] EXPRESS Language Reference Manual, ISO TC184/SC4/* WG5 N 55, Document N51, Part 11 of ISO 10303 International Standard, Jan. 1994.

[FaRS93] F. Fabret, M. Regnier, E. Simon An Adaptive Algorithm for Incremental Evaluation of Production Rules in Databases, Proc. of 19th VLDB, Dublin, Ireland, 455-466, Aug. 1993.

[FDS91] FDS Forschungszentrum des Deutschen Schiffbaus Innovative Anwendungen der Informationstechnik im Schiffbau, Abschlußbericht zur Projektdefinitionsphase, 1991.

[Fisc94] R. Fischbach, Architektur-CAD ist anders - Einzelstück, iX, Vol. 5, 1994.

[Frie93] G. Friedrich Model-Based Diagnosis and Repair, AI Communications, Vol. 6, No. 3/4, 187-206, Sept./Oct. 1993.

[GaDi94a] S. Gatziu, K.R. Dittrich SAMOS: An Active Object-oriented Database System, IEEE Quarterly Bulletin on Data Engineering, 15(1-4), 23-26, Dec. 1992.

[GaDi94b] S. Gatziu, K.R. Dittrich Events in an Active Object-Oriented Database System, Proc. 1st Int. Workshop on Rules in Database Systems, Edinburgh, 23-39, Aug. 1994.

[GeJa91] N. Gehani, H.V. Jagadish Ode as an Active Database: Constraints and Triggers, Proc. of 17th VLDB, Barcelona, 327-336, Sept. 1991.

[GeJS92] N. Gehani, H.V. Jagadish, O. Shmueli Composite Event Specification in Active Databases: Model and Implementation, Proc. of 18th VLDB, Vancouver, Britisch Columbia, Canada, 327-338, Aug. 1992.

[GL88] Germanischer Lloyd Vorschriften für Klassifikation und Bau von stählernen Seeschiffen, Germanischer Lloyd, Hamburg, Okt. 1988.

[Hall74] USM bausysteme Haller, midi 1000, Tragwerk Planungsgrundlagen, USM bausysteme Haller, 1974 .

[Hall85] F. Haller Armilla - ein Installationsmodell, Institut für Industrielle Bauproduktion, Universität Karlsruhe, 1985.

[Hans92] E.N. Hanson Rule Condition Testing and Action Execution in Ariel, Proc. ACM SIGMOD Conf. on Management of Data, San Diego, California, 4958, June 1992.

[Herz94] U. Herzog Eine Basis für schnelle Konsistenztests, Tagungsband 6. Workshop "Grundlagen von Datenbanken", Bad Helmstedt, 61-65, Sept. 1994.

[HFH+93] F. Haller, K. Friedrichs, V. Hovestadt, P. C. Lockemann, J. A. Mülle, R. Sturm The Design Navigator, Proc. 5th Int. Conf. Computing in Civil and Building Engineering, Anaheim, California, 335-340, 1993.

[HOAI91] HOAI: Verordnung über die Honorare für Leistungen der Architekten und Ingenieure, Fassung der vierten Änderungs-Verordnung, Stand 1.1.1991, Dïsseldorf, 1991. 
[HoMS94] V. Hovestadt, J. A. Mülle, R. Sturm ArchE - Datenbankunterstützte Architektur-Entwurfsumgebung: Eine Anforderungsanalyse, Technical Report No. 23, University Karlsruhe, 1994.

[IEEE92] Bulletin of the Technical Committee on Data Engineering, Special Issue on Active Databases, Vol. 15, No. 1-4, Dec. 1992.

[IGR93] Integrated Generic Resources: Geometric and Topological Representation, TCP 184/SC4/WG3, part 42 of ISO 10303 International Standard, May 1993.

[Jaeg94] U. Jaeger, Annotated Bibliography on Active Databases, HumboldtUniversität zu Berlin, Dec. 1994, to be published.

[Jasp94] H. Jasper Active Databases for Active Repositories, Proc. 10th Int. IEEE Conf. on Data Engineering, Houston, Texas, Feb. 1994.

[JBK+93] S. Jablonski, S. Barthel, T. Kirsche, T. Rödinger, H. Schuster, H. Wedekind Datenbankunterstützung für kooperative Gruppenarbeit, it+ti, 35(1993)1, 34-44, 1993.

[KiLS91] W. Kim, Y.-J. Lee, J. Seo An Overview of Integrity Management in Object-Oriented Databases, IEEE Bulletin of the Computer Society Technical Committe on Data Engineering, Vol. 14, No. 2, 38-42, June 1991.

[KoDM88] A. Kotz, K.R. Dittrich, J. Mülle Supporting Semantic Rules by a Generalized Event/Trigger Mechanism, Proc. Int. Conf. on Extending Database Techn., Venice, 76-91, 1988.

[LAB+85] P. C. Lockemann, M. Adams, M. Beuer, K. R. Dittrich B. Ferkinghoff, W. Gotthard, A. M. Kotz, R. P. Liedtke, B. Lüke, J. A. Mülle Anforderungen technischer Anwendungen an Datenbanksysteme, Proc. der GIFachtagung Datenbanken für Büro, Technik und Wissenschaft (BTW), Springer-Verlag, Heidelberg, Informatik Fachberichte Nr. 94, 1-26, 1985.

[LMSH94] P. C. Lockemann, J. A. Mülle, R. Sturm, V. Hovestadt Modeling and Integrating Design Data from Experts in a CAAD-Environment, 1st. European Conference on Product and Process Modelling in Building Industry, Dresden, Germany, 1994.

[McDa89] D. McCarthy, U. Dayal The Architecture of an Active Data Base Management System, Proc. 1989 ACM SIGMOD Conf. on Management of Data, Portland, Oregon, 215-224, June 1989.

[MoLo91] G. Moerkotte, P. C. Lockemann Reactive Consistency Control in Deductive Databases, ACM Transactions on Database Systems, Vol. 16, No. 4, 670-702, Dec. 1991.

[QiSm93] X.Qian, D.R. Smith Integrity Constraint Reformulation For Efficient Validation Proc. of 19th VLDB, Dublin, Ireland, 417-425, Aug. 1993.

[RuSK91] M. Rusinkiewicz, A. Sheth, G. Karabatis Specifying Interdatabase Dependencies in a Multidatabase Environment, COMPUTER, 46-53, Dec. 1991.

[SGKG86] P. Suter, R. Gfeller, N. Kohler, J. van Glist. Haustechnik in der Integralen Planung, Impulsprogramm Haustechnik 1986. Bundesamt für Konjunkturfragen, Bern, 1986.

[SrHT90] J. Srivastava, K.-W. Hwang, J.S.E. Tan Parallelism in Database Production Systems Proc. 6th Int. Conf. on Data Engineering, LA, 121-128, Feb. 1990 .

[SPAM91] U. Schreier, H. Pirahesh, R. Agrawal, C. Mohan Alert: An Architecture for Transforming a Passive DBMS into an Active DBMS, Proc. of 17 th VLDB, Barcelona, 469-478, Sept. 1991. 
[StHP91] M. Stonebraker, M. Hearst, S. Potamianos A Commentary on the Postgres Rules System, SIGMOD Record 18, 14(7):897-909, Sept. 1989.

[StLo94] R. Sturm, P. C. Lockemann Bereichsdynamische Konsistenzüberwachung in Architekturdatenbanken, Technical Report No. 24, University Karlsruhe, 1994.

[VoKe91] M. H. van der Voort, M. L. Kersten, Facets of database triggers, Centrum voor Wiskunde en Informatica; Computer Science/Department of Algorithmics and Architecture; Report CS-R9122, March 1991.

[Wido92] J. Widom The Starburst Rule System: Language Design, Implementation, and Applications, In [IEEE92], 15-18.

[WiHS94] H.-J. Widmer, A. Hirsch, Th. Siodla Neuartige Organisationsformen in der Konstruktion als Basis für die arbeitsorientierte Gestaltung des CADReferenzmodells Proc. CAD'94 "Produktdatenmodellierung und Prozeßmodellierung als Grundlage neuer CAD-Systeme", Fachtagung der GI, Paderborn, 295-316, März 1994. 